\title{
Obesity Alters Endoxifen Plasma Levels in Young Breast Cancer Patients: A Pharmacometric Simulation Approach
}

\author{
Anna Mueller-Schoell ${ }^{1,2}$ (D) , Lena Klopp-Schulze ${ }^{1}$, Werner Schroth ${ }^{3,4}$, Thomas Mürdter $^{3,4}$, Robin Michelet $^{1}$ (D), \\ Hiltrud Brauch $^{3,4,5}$ (D), Wilhelm Huisinga ${ }^{6}$, Markus Joerger ${ }^{7}$, Patrick Neven ${ }^{8}$, Stijn L.W. Koolen ${ }^{9}$, \\ Ron H.J. Mathijssen ${ }^{9}$, Ellen Copson ${ }^{10,11}$, Diana Eccles ${ }^{10,11}$, Sylvia Chen ${ }^{12}$, Balram Chowbay ${ }^{12,13,14}$, \\ Arafat Tfayli $^{15}$, Nathalie K. Zgheib ${ }^{16}$ (D) , Matthias Schwab ${ }^{3,5,17}$ (D) and Charlotte Kloft ${ }^{1, *}$
}

Endoxifen is one of the most important metabolites of the prodrug tamoxifen. High interindividual variability in endoxifen steady-state concentrations $\left(\mathrm{C}_{\mathrm{SS}, \mathrm{min} \mathrm{ENDX}}\right)$ is observed under tamoxifen standard dosing and patients with breast cancer who do not reach endoxifen concentrations above a proposed therapeutic threshold of $5.97 \mathrm{ng} / \mathrm{mL}$ may be at a $26 \%$ higher recurrence risk compared with patients with endoxifen concentrations exceeding this value. In this investigation, 10 clinical tamoxifen studies were pooled (1,388 patients) to investigate influential factors on $\mathrm{C}_{\mathrm{SS} \text {,min ENDX }}$ using nonlinear mixed-effects modeling. Age and body weight were found to significantly impact $\mathrm{C}_{\mathrm{SS} \text {, min }}$ ENDX in addition to CYP2D6 phenotype. Compared with postmenopausal patients, premenopausal patients had a $30 \%$ higher risk for subtarget $\mathrm{C}_{\mathrm{Ss} \text {, min ENDX }}$ at tamoxifen $20 \mathrm{mg}$ per day. In treatment simulations for distinct patient subpopulations, young overweight patients had a 3.1-13.8-fold higher risk for subtarget $\mathrm{C}_{\mathrm{SS}, \min \mathrm{ENDX}}$ compared with elderly low-weight patients. Considering ever-rising obesity rates and the clinical importance of tamoxifen for premenopausal patients, this subpopulation may benefit most from individualized tamoxifen dosing.

\section{Study Highlights}

\author{
WHAT IS THE CURRENT KNOWLEDGE ON THE \\ TOPIC? \\ $\square$ Large interindividual variability in concentrations of ta- \\ moxifen's most active metabolite endoxifen is observed during \\ standard breast cancer tamoxifen treatment. Minimal steady- \\ state endoxifen concentrations have been suggested below \\ which the risk for breast cancer recurrence and mortality is \\ increased. The influence of age and body weight on endoxifen \\ concentrations is not well-established. \\ WHAT QUESTION DID THIS STUDY ADDRESS? \\ $\checkmark$ What is the quantitative impact of age and body weight on \\ the pharmacokinetics (PKs) of tamoxifen and endoxifen be- \\ yond the patients' genetically determined CYP2D6 tamoxifen \\ metabolizer capacity?
}

WHAT DOES THIS STUDY ADD TO OUR KNOWLEDGE?

$\square$ Age and body weight contribute to the PKs of tamoxifen and endoxifen in that young and overweight patients are at increased risk to not achieve sufficient endoxifen concentrations. HOW MIGHT THIS CHANGE CLINICAL PHARMACOLOGY OR TRANSLATIONAL SCIENCE?

$\square$ Obese premenopausal patients may benefit most from individualized tamoxifen dosing, particularly in the case of an intact genetically determined tamoxifen drug metabolism. If their CYP2D6 function is impaired, alternative endocrine treatment of ovarian function suppression combined with aromatase inhibitors should be considered.

\footnotetext{
${ }^{1}$ Department of Clinical Pharmacy and Biochemistry, Institute of Pharmacy, Freie Universitaet Berlin, Berlin, Germany; ${ }^{2}$ Graduate Research Training Program PharMetrX, Berlin/Potsdam, Germany; ${ }^{3}$ Dr. Margarete Fischer-Bosch Institute of Clinical Pharmacology, Stuttgart, Germany; ${ }^{4}$ University of Tübingen, Tübingen, Germany; ${ }^{5}$ German Cancer Consortium (DKTK) and of German Cancer Research Center (DKFZ), Heidelberg, Germany; ${ }^{6}$ Institute of Mathematics, University of Potsdam, Potsdam, Germany; ${ }^{7}$ Department of Medical Oncology and Haematology, Cantonal Hospital, St. Gallen, Switzerland; ${ }^{8}$ Vesalius Research Center - VIB, University Hospitals Leuven, KU Leuven-University of Leuven, Leuven, Belgium; ${ }^{9}$ Department of Medical Oncology, Erasmus MC Cancer Institute, Rotterdam, The Netherlands; ${ }^{10}$ Cancer Sciences Academic Unit and University of Southampton Clinical Trials Unit, Faculty of Medicine, University of Southampton, Southampton, UK; ${ }^{11}$ University Hospital Southampton NHS Foundation Trust, Southampton, UK; ${ }^{12}$ Clinical Pharmacology Laboratory, Division of Cellular \& Molecular Research, Humphrey Oei Institute of Cancer Research, National Cancer Centre Singapore, Singapore; ${ }^{13}$ Center for Clinician-Scientist Development, Duke-NUS Medical School, Singapore; ${ }^{14}$ SingHealth Clinical Pharmacology, SingHealth, Singapore; ${ }^{15}$ Hematology-Oncology Division, Department of Internal Medicine, Faculty of Medicine, American University of Beirut, Beirut, Lebanon; ${ }^{16}$ Department of Pharmacology and Toxicology, Faculty of Medicine, American University of Beirut, Beirut, Lebanon; ${ }^{17}$ Departments of Clinical Pharmacology, Pharmacy and Biochemistry, University Tübingen, Tübingen, Germany. *Correspondence: Charlotte Kloft (charlotte.kloft@fu-berlin.de)
} 
Tamoxifen treatment for 5-10 years is widely used in premenopausal and an option in postmenopausal patients with estrogen receptor positive breast cancer. ${ }^{1,2}$ During its use for $>40$ years, a 5 -year adjuvant tamoxifen treatment has been proven to effectively reduce breast cancer recurrence by around $30 \%$ in the first 15 years of therapy. ${ }^{3}$ Tamoxifen is extensively metabolized and considered to be the pro-drug to its 100 -fold more active metabolite endoxifen. ${ }^{4,5}$

Several polymorphic enzymes, such as CYP2D6, CYP2C9, CYP2C19, CYP3A5, sulfotransferases, and UDPglucuronosyltransferases, are involved in tamoxifen metabolism $^{5,6}$ and consequently large interindividual variability (IIV) in endoxifen minimum concentrations at steady-state $\left(\mathrm{C}_{\mathrm{SS} \text {,min }}\right.$ ENDX) has been observed under tamoxifen standard dosing (20 mg once daily (q.d.))..$^{7-9}$ CYP2D6 is especially important for endoxifen formation and patients with impaired or no CYP2D6 activity have shown an increased risk for subtarget $\mathrm{C}_{S S \text {, min ENDX. }}{ }^{8-11}$ Regarding a putative therapeutic threshold concentration, Madlensky et al. reported that patients with $\mathrm{C}_{S S, \text { min ENDX }}<5.97 \mathrm{ng} / \mathrm{mL}$ had a $26 \%$ higher breast cancer recurrence rate compared with patients with $\mathrm{C}_{S S, \min \text { ENDX }}$ above this threshold (recurrence rates $16 \%$ vs. $10.1-14.7 \%$ ). ${ }^{9}$ This difference is similar to the reported $30 \%$ relative reduction in breast cancer recurrence rates, when postmenopausal patients receive aromatase inhibitors instead of tamoxifen. ${ }^{12}$ The aforementioned target concentration was later supported by Saladores $e t$ al. for premenopausal patients. ${ }^{8}$ Other studies failed to find the described relationship between $\mathrm{C}_{S S, \text { min ENDX }}$ and/or CYP2D6 and treatment outcome, ${ }^{13-15}$ which might, in part, be due to heterogeneous patient populations, study designs, DNA source used for CYP2D6 genotype determination, ${ }^{7,16}$ and insufficient power to detect the relationships. ${ }^{17,18}$ Accordingly, the efficacy of breast cancer tamoxifen treatment may be influenced by the proposed target threshold, however, nongenetic factors beyond CYP2D 6 functionality, influencing the pharmacokinetics (PKs) of tamoxifen and endoxifen may play a role. Of those, a positive correlation between patient age and tamoxifen concentrations has been described in literature ${ }^{19-21}$ and was later quantified and found to be clinically relevant in a PK analysis using nonlinear mixed-effects modeling. ${ }^{22}$ Furthermore, increased body weight or body mass index (BMI) have been associated with decreased concentrations of tamoxifen and its primarily lipophilic metabolites ${ }^{8,9,19,23}$ and worse clinical outcome. ${ }^{24,25}$ However, the impact of body weight on $\mathrm{C}_{S S, \text { min ENDX }}$ has never been quantified.

In this work, we applied mathematical modeling and simulations to quantify the influence of age and body weight on $\mathrm{C}_{S S, \min E N D X}$ in patients treated with tamoxifen and report a patient subpopulation at risk for subtarget endoxifen concentrations.

\section{METHODS}

\section{Clinical study database}

A large tamoxifen clinical study dataset was compiled by pooling data from 10 clinical studies. Studies $1-6^{26-30}$ (referred to as "development dataset," previously pooled at the Freie Universitaet Berlin, Germany) and studies $7-10^{8,10}$ (referred to as "evaluation dataset," previously pooled at the Dr. Margarete Fischer-Bosch Institute of Clinical Pharmacology in Stuttgart, Germany) are described in detail elsewhere. ${ }^{10,22}$ All studies were conducted in accordance with the ethical standards of the Declaration of Helsinki and had been approved by the respective ethics committees.

The pooled dataset comprised demographic, PK and pharmacogenetic data, and tamoxifen and endoxifen steady-state (SS) plasma concentrations in 1,388 female patients with breast cancer receiving $20 \mathrm{mg}(n=1,373)$ or $40 \mathrm{mg}(n=15)$ tamoxifen once daily (q.d.; Table 1). Tamoxifen and endoxifen concentrations were analyzed in plasma or serum using liquid chromatography linked with tandem mass spectrometry (detailed information in Supplementary Tables S1 and S2). As studies were conducted independently from each other, no cross-validation between laboratories was performed. Patients receiving strong CYP2D6 inhibitors or CYP3A4 inducers and patients who had not yet reached $S S$ were excluded from the development dataset $(n=16)$ prior to pooling.

According to the Clinical Pharmacogenetics Implementation Consortium (CPIC) guideline for CYP2D6 and Tamoxifen Therapy, patients were assigned CYP2D6 activity scores (AS) based on their CYP2D6 diplotypes. ${ }^{31}$ Genotype-predicted phenotype assignment was as follows: (i) AS of 0 refers to poor metabolizers (gPM), (ii) AS of $0.5-1$ refers to intermediate metabolizers (gIM), and (iii) AS of $\geq 1.5$ refers to normal metabolizers (gNM; including ultrarapid-metabolizers $($ AS $>2)) .{ }^{32}$ For patients with missing genotype information $(n=39$, 2.81\%) the CYP2D6 wildtype (AS $=2)$ as most frequent CYP2D6 AS was imputed.

Menopausal status had not been reported in the development dataset and was imputed for patients with missing information based on the intersection of the age densities for premenopausal and postmenopausal patients in the evaluation dataset ( 52 years, in line with the definition used by the North American Menopause Society $\left.{ }^{33}\right)$. The development dataset included white $(n=433)$ and African $(n=2)$ patients, whereas the evaluation dataset included premenopausal and postmenopausal white patients $(n=681)$ and premenopausal Africans $(n=12)$, Middle-Eastern Arabs $(n=77)$, Asians $(n=153)$, and Indians $(n=12)$. For patients without reported ethnicity $(n=14,1.01 \%)$, white ethnicity, as the most frequent, was imputed.

\section{Joint parent-metabolite PK model of tamoxifen and endoxifen, and external model evaluation}

The joint parent-metabolite nonlinear mixed-effects modeling PK model of tamoxifen and endoxifen developed using the development dataset $^{22}$ was externally evaluated using the evaluation dataset. A one-compartment model-parameterized in terms of relative clearances $(\mathrm{CL} / \mathrm{F})$ and volumes of distributions, with first-order absorption with lag time for tamoxifen-was linked to an endoxifen one-compartment model via a linear first-order formation process (CL23/F). Elimination of both tamoxifen and endoxifen (CL20/F and CL30/F, respectively) were described as linear first-order processes. Parameter values for endoxifen apparent clearance (CL30/F) and endoxifen apparent volume of distribution $\left(\mathrm{V}_{\mathrm{ENDX}} / \mathrm{F}\right)$ were adopted from a study in which endoxifen had been administered as a single compound. ${ }^{34}$ IIV parameters were estimated for both tamoxifen clearance and endoxifen formation (CL20/F and CL23/F, respectively), whereas interoccasion variability was not considered, as only one PK sample per patient was available in the evaluation dataset. CYP2D6 AS and age as significant covariates on endoxifen formation and tamoxifen clearance, respectively, were implemented as proportional and power functions, respectively.

Based on the final estimates using the development dataset, tamoxifen and endoxifen concentrations were predicted for the evaluation dataset and compared with observed concentrations. Mean absolute prediction errors and mean prediction errors were calculated to assess precision and 
bias, respectively. ${ }^{35}$ Finally, model parameters, except absorption parameters, which were fixed to the estimates obtained during model development as the evaluation dataset contained $\mathrm{C}_{S \mathrm{~S} \text {,min }}$ only, were re-estimated using the pooled dataset and compared with previously estimated parameters using the development dataset.

\section{Extensive covariate analysis and final model development}

Patient characteristics were preselected for the extensive covariate analysis based on physiological plausibility, previous literature reports, and sufficient information in the pooled dataset. A relationship between increasing age and decreasing tamoxifen clearance had been reported ${ }^{21}$ and was supported by our previous analysis using the development dataset. ${ }^{22}$ To evaluate this relationship in the pooled dataset and test for differences between both datasets, $\mathrm{C}_{S \mathrm{~S} \text { min FNDX }}$ were compared between premenopausal and postmenopausal patients receiving $20 \mathrm{mg}$ tamoxifen q.d. in the development $(n=435)$ and evaluation $(n=935)$ datasets, respectively. Based on expected PK differences between ethnicities, ${ }^{36} \mathrm{C}_{\text {SS,min ENDX }}$ were additionally compared between premenopausal patients of different ethnicities in the evaluation dataset. To evaluate the contribution of varying CYP2D6 phenotype frequencies to the observed differences between ethnicities, $\mathrm{C}_{\mathrm{SS} \text {,min ENDX }}$ were compared in premenopausal patients of different ethnicities stratified for CYP2D6 phenotype.

To assess if differences between patient subpopulations were statistically significant, nonparametric Wilcoxon tests were performed. The extensive covariate analysis was based on the pooled dataset and the original PK base model. Age, CYP2D6 AS, and newly selected covariates, body weight as proportional or power function, and ethnicity as categorical, function were tested for significance on model parameters endoxifen formation (CL23/F), endoxifen clearance (CL30/F), and tamoxifen clearance (CL20/F) using stepwise covariate model-building. ${ }^{37}$ Significance criteria of $3.84(\alpha=0.05)$ and $7.88(\alpha=0.005)$ points change in objective function value were applied in the inclusion and exclusion steps, respectively. Finally, goodness-of-fit plots were created to assess model performance.

Treatment simulations for different patient subpopulations Applying the updated joint parent-metabolite PK model with its final parameter estimates, treatment simulations were performed to investigate the impact of age and body weight on achieving target $\mathrm{C}_{S S \text {, min ENDX }}$ under tamoxifen standard dosing. In two separate simulation study set-ups, 14 large virtual patient populations $(n=10,000$ each) with CYP2D6 AS frequencies extrapolated from the pooled dataset and different age and body weight ranges or combinations thereof were generated:

Study set-up 1: Endoxifen subtarget concentrations for subpopulations with different age and body weight distributions. Study set-up 1 (SU1) was based on the observed distributions of age and body weight in the pooled dataset. Achievement of target $\mathrm{C}_{\mathrm{SS} \text {,min ENDX }}$ was compared between patients with low or high covariate values (less than the first quartile and greater than the third quartile, respectively) and patients with covariate values in the interquartile range of the covariate value distribution in the pooled dataset ("reference subpopulation"; in total: 7 patient populations; Table 2). Specifically, for each virtual patient, an age and body weight value were sampled independently with replacement from the respective section (e.g., less than the first quartile) of the covariate value distribution in the pooled dataset.

Study set-up 2: Endoxifen subtarget concentrations for subpopulations with extreme age and body weight values. In study set-up 2 (SU2), $\mathrm{C}_{\mathrm{SS} \text {, min ENDX }}$ target attainment was compared between virtual patients with minimum or maximum covariate values and patients with median covariate values in the pooled dataset ("reference subpopulation"; in total: 7 patient populations; Table 2).

Table 1 Clinical study and population characteristics of the development, evaluation, and pooled dataset at baseline

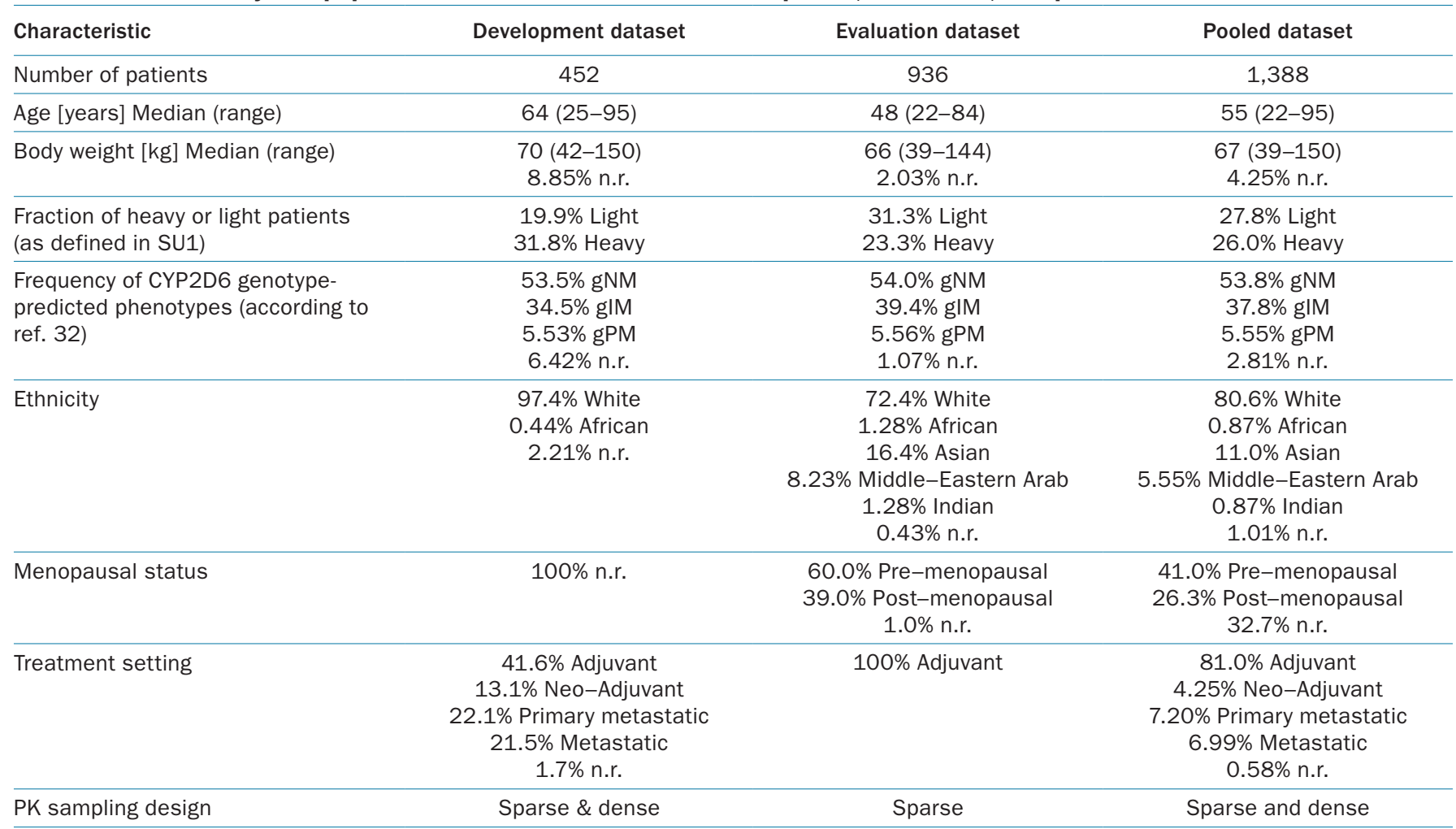

gNM, gIM, gPM, genotype-predicted CYP2D6 normal (including ultrarapid), intermediate and poor metabolizer; n.r., not reported; PK, pharmacokinetic(s); SU1, study setup 1 . 
Table 2 Covariate values used in simulating 14 different patient subpopulations (seven per study-setup) (see main text for detailed explanations of study set-ups 1 and 2)

\begin{tabular}{|c|c|c|c|c|}
\hline Subpopulation ( $n=10,000$ each) & \multicolumn{2}{|c|}{ Study-setup 1} & \multicolumn{2}{|c|}{ Study-setup 2} \\
\hline Heavy young & 22-39 (<Q1) & $77-150(>Q 3)$ & 22 (Min.) & 150 (Max.) \\
\hline Young & 22-39 (<Q1) & 60-76 (IQR) & 22 (Min.) & 68 (Med.) \\
\hline Heavy & 40-65 (IQR) & $77-150(>Q 3)$ & 55 (Med.) & 150 (Max.) \\
\hline IQR/Median (Reference) & 40-65 (IQR) & 60-76 (IQR) & 55 (Med.) & 68 (Med.) \\
\hline Light & $39-60(<Q 1)$ & 40-65 (IQR) & 55 (Med.) & 39 (Min.) \\
\hline Light elderly & $66-95$ (>Q3) & $39-60(<Q 1)$ & 95 (Max.) & 39 (Min.) \\
\hline
\end{tabular}

Contents of the brackets indicate which part of the covariate distribution in the pooled dataset is represented.

IQR, interquartile range; Max., maximum; Med., median, Min., minimum; $Q_{x}$, Quartile with $x=1-3$.

To account for parameter uncertainty, 1,000 simulations using bootstrapped parameter sets were performed for each subpopulation and the 50th, 5th, and 95th percentiles were used to determine medians and $90 \%$ confidence intervals (CIs), respectively, of (i) the fraction of patients of the respective subpopulation at risk for $\mathrm{C}_{S S \text {, min }}$ ENDX target nonattainment, (ii) the absolute change in risk compared with the respective reference subpopulation, (iii) the relative change in risk compared with the respective reference subpopulation, and (iv) the number needed to treat/harm (NNT/NNH), defined as 1 divided by the absolute change in risk, compared with the respective reference subpopulation. Thus, the ratio described the NNH if the absolute change in risk was positive, and the NNT if the absolute change in risk was negative. Finally, for the two patient populations that had shown the highest risk for subtarget $\mathrm{C}_{S S \text {, min ENDX }}$ in SU1 and SU2, $\mathrm{C}_{S S \text {, min }}$ ENDX at alternative daily tamoxifen doses of $40 \mathrm{mg}$ and $60 \mathrm{mg}$ were simulated and medians and $90 \%$ CIs of the fractions of patients at risk for subtarget $\mathrm{C}_{\mathrm{SS} \text {, min ENDX }}$ were calculated.

\section{RESULTS}

\section{External model evaluation}

The original joint-parent metabolite population PK model of tamoxifen and endoxifen performed well for the evaluation dataset: mean prediction errors indicated a low bias for tamoxifen $(-13.9 \mathrm{ng} / \mathrm{mL})$ and a minimal bias for endoxifen $(-0.923 \mathrm{ng} / \mathrm{mL})$. Precision was acceptable for both tamoxifen and endoxifen, as indicated by MAPEs $<8 \%$ (7.62\% and 6.29\%, respectively). ${ }^{38}$ After parameter re-estimation using the pooled dataset, all fixed (structural and covariate) parameter estimates remained comparable except the tamoxifen clearance CL20/F for a typical (AS 2, median age 55 years) patient (development dataset: $6.51 \mathrm{~L} / \mathrm{h}$ (2.4\% relative standard error (RSE)), pooled dataset: $5.08 \mathrm{~L} / \mathrm{h}(1.1 \% \mathrm{RSE})$, and the exponent for the typical age effect on the tamoxifen clearance (development dataset: $-0.844(10.0 \%)$, pooled dataset: $-0.148(24.0 \%))$. Furthermore, estimated IIV values on CL20/F and CL23/F were slightly lower ( $40.4 \%$ vs. $41.5 \%$ and $46.1 \%$ vs. $49.2 \%$, respectively) for the pooled dataset compared with the development dataset.

\section{Extended covariate analysis and final model development}

A significant difference between $\mathrm{C}_{S S \text {,min ENDX }}$ in premenopausal $(n=67)$ and postmenopausal $(n=368)$ patients was observed in the development dataset ( $97.4 \%$ white patients; Table S3): whereas $29.9 \%$ of premenopausal patients showed subtarget $\mathrm{C}_{\text {SS, min ENDX }}<5.97 \mathrm{ng} / \mathrm{mL}$, it was only $20.1 \%$ of postmenopausal patients (Table 3 ). Conversely, in the evaluation dataset, with $18.8 \%$ and $18.0 \%$ of patients with subtarget $\mathrm{C}_{\mathrm{SS} \text {,min ENDX }}$ (Table 3), there was no difference in $\mathrm{C}_{\mathrm{SS} \text {, min }}$ ENDX between premenopausal $(n=568)$ and postmenopausal $(n=367)$ patients (Table S3). However, after stratifying patients in the evaluation dataset for their ethnicity, a highly significant difference between $\mathrm{C}_{S S \text {, min ENDX }}$ in premenopausal and postmenopausal white patients became apparent (Tables 3 and S3). Furthermore, there were large differences between $\mathrm{C}_{\mathrm{SS} \text {,min } \mathrm{ENDX}}$, ascending from premenopausal Africans, whites, Middle-Eastern Arab, and Asian to Indian patients (Table S3). Indians, Asians, and Middle-Eastern Arabs showed the lowest number of patients with subtarget $\mathrm{C}_{S S \text {, min ENDX }}(0 \%, 5.8 \%$, and $13.0 \%$, respectively) whereas Africans and white patients showed the highest $(50.0 \%$ and $26.1 \%$, respectively). Of note, relative risk reductions due to transition from premenopause to postmenopause were $32.8 \%$ in the development dataset $(n=433$ whites, $n=2$ Africans), $4.26 \%$ for the evaluation dataset without stratification for ethnicity $(n=935)$ and $31.0 \%$ for white patients in the evaluation dataset $(n=681$; no further analysis was possible as no data from postmenopausal patients of other ethnicities were available). Upon stratification for CYP2D6 phenotype, the differences in $\mathrm{C}_{S S \text {, min ENDX }}$ between premenopausal patients of different ethnicities remained. Further exploratory analyses revealed a correlation between body weight and ethnicity in the evaluation dataset. Body weight was highest in premenopausal Middle-Eastern Arabs, followed by whites, Africans, Indians, and Asians (Table S4). Furthermore, patients of ethnicities with low body weights demonstrated a lower risk for subtarget $\mathrm{C}_{S S \text {, min ENDX }}$ compared with patients of ethnicities with high body weights (Figures S1 and S2). Subsequently, both ethnicity and body weight were tested for significance on CL20/F, CL23/F, and CL30/F in the extended covariate analysis.

Covariate relationships of CYP2D6 AS on CL23/F (categorical), age and body weight on CL20/F (both power functions), and ethnicity on CL20/F (categorical) were all significant in univariate analyses. Including ethnicity on CL20/F in addition to body weight, however, did not further improve model predictions. Due 

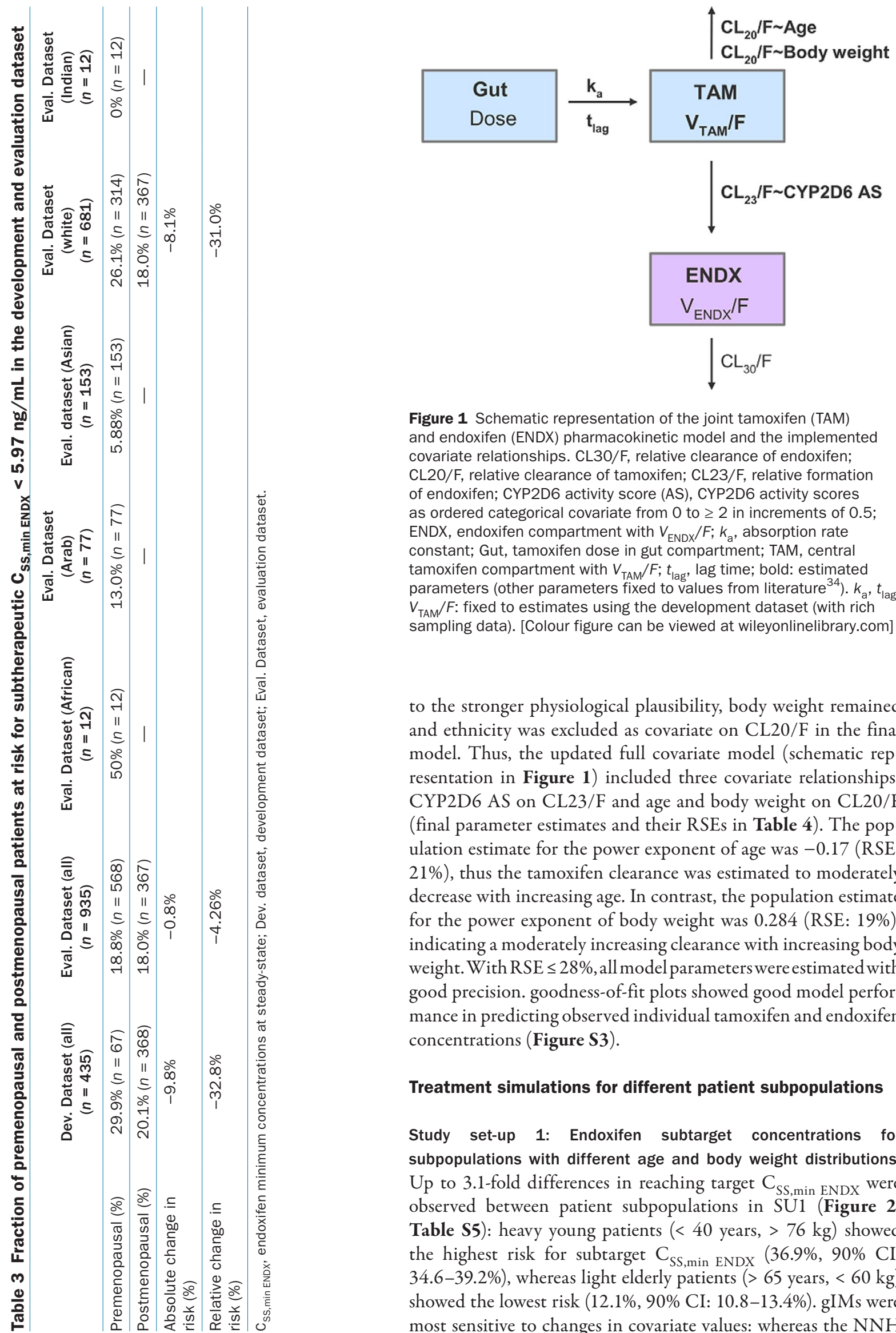

Figure 1 Schematic representation of the joint tamoxifen (TAM) and endoxifen (ENDX) pharmacokinetic model and the implemented covariate relationships. CL30/F, relative clearance of endoxifen; CL20/F, relative clearance of tamoxifen; CL23/F, relative formation of endoxifen; CYP2D6 activity score (AS), CYP2D6 activity scores as ordered categorical covariate from 0 to $\geq 2$ in increments of 0.5 ; ENDX, endoxifen compartment with $V_{\text {ENDX }} / F ; k_{a}$, absorption rate constant; Gut, tamoxifen dose in gut compartment; TAM, central tamoxifen compartment with $V_{\text {TAM }} / F$; $t_{\text {lag }}$, lag time; bold: estimated parameters (other parameters fixed to values from literature $\left.{ }^{34}\right) . k_{\mathrm{a}}, t_{\text {lag }}$, $V_{\text {TAM }} / F$ : fixed to estimates using the development dataset (with rich sampling data). [Colour figure can be viewed at wileyonlinelibrary.com]

to the stronger physiological plausibility, body weight remained and ethnicity was excluded as covariate on CL20/F in the final model. Thus, the updated full covariate model (schematic representation in Figure 1) included three covariate relationships: CYP2D6 AS on CL23/F and age and body weight on CL20/F (final parameter estimates and their RSEs in Table 4). The population estimate for the power exponent of age was -0.17 (RSE: $21 \%$ ), thus the tamoxifen clearance was estimated to moderately decrease with increasing age. In contrast, the population estimate for the power exponent of body weight was 0.284 (RSE: 19\%), indicating a moderately increasing clearance with increasing body weight. With RSE $\leq 28 \%$, all model parameters were estimated with good precision. goodness-of-fit plots showed good model performance in predicting observed individual tamoxifen and endoxifen concentrations (Figure S3).

Treatment simulations for different patient subpopulations

Study set-up 1: Endoxifen subtarget concentrations for subpopulations with different age and body weight distributions. Up to 3.1-fold differences in reaching target $\mathrm{C}_{S S \text {,min ENDX }}$ were observed between patient subpopulations in SU1 (Figure 2, Table S5): heavy young patients (< 40 years, $>76 \mathrm{~kg}$ ) showed the highest risk for subtarget $\mathrm{C}_{S S, \min \text { ENDX }}(36.9 \%, 90 \% \mathrm{CI}$ : $34.6-39.2 \%)$, whereas light elderly patients $(>65$ years, $<60 \mathrm{~kg})$ showed the lowest risk (12.1\%, 90\% CI: 10.8-13.4\%). gIMs were most sensitive to changes in covariate values: whereas the $\mathrm{NNH}$ 
Table 4 Final parameter estimates for the updated joint parent-metabolite population pharmacokinetic model of tamoxifen and endoxifen using the pooled dataset (1,388 patients)

\begin{tabular}{|c|c|c|c|}
\hline Parameter (unit) & Parameter description & Estimate & RSE, \% \\
\hline \multicolumn{4}{|l|}{ Fixed effects } \\
\hline$k_{\mathrm{a}}(1 /$ hour $)$ & Absorption rate constant & 1.08 Fixed & \\
\hline$t_{\text {lag }}$ (hour) & Absorption lag time & 0.442 Fixed & \\
\hline $\mathrm{V}_{\mathrm{TAM}} / F(\mathrm{~L})$ & Tamoxifen apparent volume of distribution & 912 Fixed & \\
\hline CL30/F (L/hour) & Apparent endoxifen clearance & 5.10 Fixed & \\
\hline$V_{\text {ENDX }} / F(\mathrm{~L})$ & Endoxifen apparent volume of distribution & 400 Fixed & \\
\hline CL20/F (L/hour) & Apparent tamoxifen clearance & 5.07 & 1 \\
\hline CL20/F_Age ${ }^{a}$ & $\begin{array}{c}\text { Exponent for the covariate effect of age on the } \\
\text { apparent tamoxifen clearance }\end{array}$ & -0.17 & 21 \\
\hline CL20/F_Body weight ${ }^{a}$ & $\begin{array}{c}\text { Exponent for the covariate effect of body weight on } \\
\text { the apparent tamoxifen clearance }\end{array}$ & 0.284 & 19 \\
\hline CL23/F (L/hour) & Apparent endoxifen formation for an AS of 2 & 0.459 & 2 \\
\hline CL23/F_AS: $0^{b}$ & $\begin{array}{l}\text { Fractional change in the apparent endoxifen } \\
\text { formation for an AS of } 0\end{array}$ & -0.759 & 2 \\
\hline CL23/F_AS: $0.5^{b}$ & $\begin{array}{l}\text { Fractional change in the apparent endoxifen } \\
\text { formation for an AS of } 0.5\end{array}$ & -0.598 & 4 \\
\hline CL23/F_AS: $1^{b}$ & $\begin{array}{l}\text { Fractional change in the apparent endoxifen } \\
\text { formation for an AS of } 1\end{array}$ & -0.347 & 6 \\
\hline CL23/F_AS: $1.5^{\mathrm{b}}$ & $\begin{array}{l}\text { Fractional change in the apparent endoxifen } \\
\text { formation for an AS of } 1.5\end{array}$ & -0.16 & 18 \\
\hline CL23/F_AS: $2.5-3^{b}$ & $\begin{array}{l}\text { Fractional change in the apparent endoxifen } \\
\text { formation for an AS of }>2\end{array}$ & 0.302 & 28 \\
\hline \multicolumn{4}{|l|}{ Random effects } \\
\hline IIV CL20/F & $\begin{array}{l}\text { Interindividual variability in the apparent tamoxifen } \\
\text { clearance }\end{array}$ & $0.148(39.9 \% \mathrm{CV})$ & 5 \\
\hline IIV CL23/F & $\begin{array}{l}\text { Interindividual variability in the apparent endoxifen } \\
\text { clearance }\end{array}$ & $0.192(46.0 \% \mathrm{CV})$ & 5 \\
\hline RUV tamoxifen & $\begin{array}{l}\text { Residual unexplained variability in the observed } \\
\text { tamoxifen concentrations }\end{array}$ & 0.0295 (17.3\% CV) & 11 \\
\hline COV $_{\text {RUVtam-RUVendx }}$ & $\begin{array}{c}\text { Correlation between RUV tamoxifen and RUV } \\
\text { endoxifen }\end{array}$ & 0.0228 & 7.28 \\
\hline RUV endoxifen & $\begin{array}{c}\text { Residual unexplained variability in the observed } \\
\text { endoxifen concentrations }\end{array}$ & $0.037(19.4 \% \mathrm{CV})$ & 7 \\
\hline
\end{tabular}

AS, CYP2D6 activity score; CL20/F, apparent tamoxifen clearance; CL23/F, apparent endoxifen formation; CL30/F, apparent endoxifen clearance; IIV, interindividual variability; $k_{\mathrm{a}}$, absorption rate constant; RUV, residual unexplained variability; RSE, relative standard error $=\left(\right.$ standard error/estimate).100; $t_{\text {lag, }}$ absorption lag time; $V_{\text {TAM }} / F$, tamoxifen apparent volume of distribution; $V_{E N D X} / F$, endoxifen apparent volume of distribution.

${ }^{a}$ Implemented as power covariate model (detailed description in ref. 23). ${ }^{b}$ Implemented as fractional change covariate model (detailed description in ref. 23).

for heavy young gNMs and gPMs was 8 and 9 , respectively, it was 5 in gIMs (Table S6).

Study set-up 2: Endoxifen subtarget concentrations for subpopulations with extreme age and body weight values. The patterns observed in SU1 were expectedly even stronger in SU2: up to 13.8-fold differences in $\mathrm{C}_{\mathrm{SS} \text {, min ENDX }}$ target attainment were observed between heavy young ( 22 years, $150 \mathrm{~kg}$ ) and light elderly ( 95 years, $39 \mathrm{~kg}$ ) patients $(70.6 \%, 90 \%$ CI: $66.2-75.1 \%$ vs. $5.10 \%$, 90\% CI: $4.18-6.22 \%$ of patients at risk, respectively; Figure 3, Table 57 ). NNH were again lowest in heavy young patients (2 for gNMs and gIMs, 6 for gPMs; Table S8).

In both study set-ups, the impact of body weight on endoxifen $\mathrm{C}_{S S \text {, min }} \mathrm{ENDX}$ was more pronounced than the impact of age, as displayed by the lower relative risk increase in young patients (median: $+13.0 \%$, 90\% CI: 6.50-19.4\%) compared with heavy patients (median: $+58.1 \%, 90 \%$ CI: 49.8-66.8\%) when compared with the reference subpopulation in SU1.

As heavy young patients showed the highest risk for subtarget $\mathrm{C}_{S S, \min \text { ENDX }}$ in both study set-ups, $\mathrm{C}_{S S, \min \text { ENDX }}$ target attainment at $40 \mathrm{mg}$ and $60 \mathrm{mg}$ tamoxifen q.d. was assessed for this subpopulation in both SU1 and SU2 (Supplementary Figures S4 and S5).

In SU1, $40 \mathrm{mg}$ tamoxifen q.d. were sufficient to reduce the fraction of patients with subtarget $\mathrm{C}_{S S \text {, min ENDX }}$ from $36.9 \%$ to $10.6 \%$ (90\% CI: 9.44-11.8\%). This fraction varied substantially among CYP2D6 phenotypes (3.06\% for gNMs, $14.2 \%$ for gIMs, and $62.0 \%$ for gPMs). In SU2, $40 \mathrm{mg}$ tamoxifen q.d. reduced the fraction of patients with subtarget $\mathrm{C}_{\mathrm{SS} \text {, min ENDX }}$ from $70.6 \%$ to 


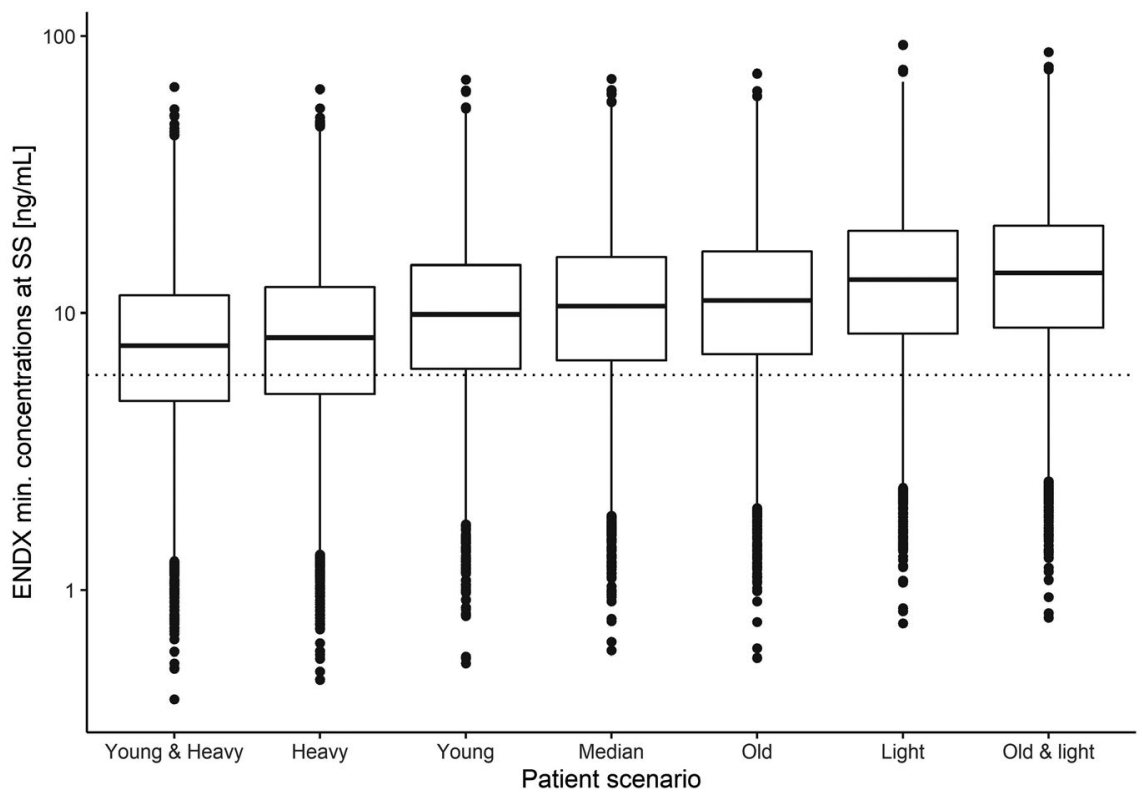

Figure 2 Patients at risk of subtarget endoxifen concentrations across patient subpopulations in study-setup 1 (see main text for further explanation) as observed in 1 of the 1,000 stochastic simulations. Simulated minimum steady-state concentrations of endoxifen (ENDX minimum concentration at steady state (SS)) in seven different patient populations with covariate characteristics as indicated on the right. Dashed horizontal line: endoxifen target threshold ${ }^{9}$; boxes: interquartile range (IQR), including median; whiskers: range from hinge to lowest/ highest value within 1.5 IQR; points: data outside whiskers.

$32.2 \%$ (90\% CI: 27.9-36.9\%). When the analysis was stratified for CYP2D6 phenotype, $18.1 \%$ of gNMs, $44.7 \%$ of gIMs, and $90.6 \%$ of gPMs remained at subtarget $\mathrm{C}_{S S, \min \text { ENDX }}$.

At $60 \mathrm{mg}$ tamoxifen q.d., $4.10 \%$ (90\% CI: $3.48-4.77 \%)$ and $15.8 \%$ (90\% CI: $13.2-18.9 \%)$ of patients still showed subtarget $\mathrm{C}_{\mathrm{SS} \text {,min ENDX }}$ in SU1 and SU2, respectively. When stratified for CYP2D 6 phenotype, $0.600 \%$ of gNMs, $4.63 \%$ of gIMs, and $36.2 \%$ of gPMs showed subtarget $\mathrm{C}_{\mathrm{SS} \text {, min ENDX }}$ in SU1 whereas it was $5.83 \%$ of gNMs, $22.3 \%$ of gIMs, and $74.4 \%$ of gPMs in SU2.

\section{DISCUSSION}

We identified young overweight patients with breast cancer as a subpopulation at increased risk for subtarget endoxifen levels during adjuvant tamoxifen treatment. This finding is of potential clinical relevance because premenopausal patients with breast cancer highly depend on the efficacy of tamoxifen given that ovarian function suppression in combination with an aromatase inhibitor can only be considered for a small portion of high-risk patients. Therefore, every effort needs to be made to increase tamoxifen efficacy, particularly in those patents with an intact CYP2D6 function for sufficient endoxifen formation.

The strength of our study is its large cohort size of 1,388 premenopausal and postmenopausal tamoxifen-treated patients with breast cancer with a wide body weight range $(39-150 \mathrm{~kg})$. This allowed us to reliably identify and quantify the influence of body weight on endoxifen SS concentration in addition to the impact of CYP2D6 function.

Of note, we informed our model parameters describing apparent endoxifen volume of distribution and clearance with previously reported values from a phase I study. ${ }^{34}$ As no demographic details were disclosed, it remains unknown whether the patients in our pooled dataset were similar to the patients studied in this cohort. Thus, future investigations using endoxifen as a single compound should add insight into these parameter values in the relevant patient population.

Using treatment simulations to investigate $\mathrm{C}_{\mathrm{SS} \text {,min ENDX }}$ in different patient subpopulations, young overweight patients were identified at highest risk for subtarget $\mathrm{C}_{S S \text {, min ENDX }}$. The design of SU1 was chosen to consider the "real-world" variability of the covariate distributions and to decrease potential bias of the simulation results due to extreme values observed in the pooled dataset. In contrast, SU2 assessed ultimate best-case and worst-case scenarios, as could be expected considering the covariate values observed in the pooled dataset. The large number of 10,000 patients for each subpopulation was used to represent the distribution of CYP2D6 phenotypes observed in "real-world" populations and allowed the generation of sufficient numbers of virtual patients with rare CYP2D6 genotypes in each subpopulation. Furthermore, it allowed to represent the high IIV observed in real-world data. The large number of 1,000 simulations with bootstrapped parameter sets for each subpopulation allowed to additionally determine CIs for the fractions of patients at risk.

The large size of our study dataset allowed us to revise and update the previously described relationship between increasing age and decreasing tamoxifen clearance. ${ }^{22}$ At first sight, this relationship was far less pronounced in the evaluation dataset compared with the development dataset indicated by a higher (less negative) power exponent in the covariate relationship of tamoxifen clearance and age. Even though bioanalytical laboratories were not cross-validated and the validated analytical methods differed between some studies, no major differences in measured concentrations, which could have explained this finding, were observed between both datasets. The difference can rather be explained 


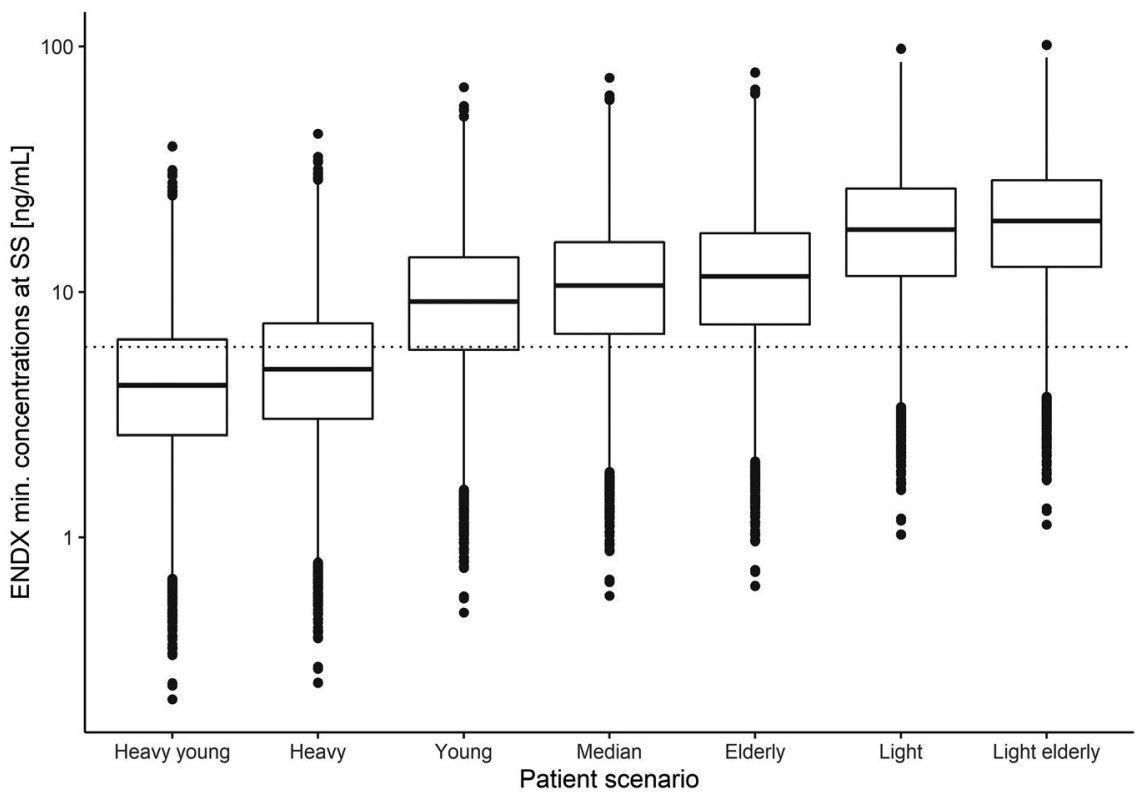

Figure 3 Patients at risk of subtarget endoxifen concentrations across patient subpopulations in study-setup 2 (see main text for further explanation) as observed in 1 of the 1,000 stochastic simulations. Simulated minimum steady-state (SS) concentrations of endoxifen (ENDX minimum concentration at SS) in seven different patient populations with covariate characteristics as indicated on the right. Dashed horizontal line: endoxifen target threshold ${ }^{9}$; boxes: interquartile range (IQR), including median; whiskers: range from hinge to lowest/highest value within 1.5 IQR; points: data outside whiskers.

by different body weight distributions. Although body weight was similar in premenopausal and postmenopausal patients in the development dataset (Table S4), it was significantly lower in premenopausal compared with postmenopausal patients in the evaluation dataset $(P<0.001)$. The latter might be explained by differences in ethnicities and cultural background. Especially Asian and Indian premenopausal patients had lower body weights compared with white individuals, who were the only ethnic group in postmenopausal patients.

Thus, the opposing influences of low body weight and young age on the tamoxifen clearance could have masked each other in the evaluation dataset. Supporting this hypothesis, relative risk reductions due to the transition from premenopause to postmenopause were similar in white patients of both datasets $(32.8 \%$ in the development dataset, and $31.0 \%$ in the evaluation dataset). Physiological explanations for our finding of decreased tamoxifen and endoxifen plasma concentrations in patients with high body weight include either (i) an increased clearance due to increased body weight causing an increased liver size and function, ${ }^{39}$ or (ii) an increased distribution of the more lipophilic compound tamoxifen into fat tissue ( $\log P$-values: 7.1, 6.7, and 6.3 for tamoxifen, $N$ desmethyltamoxifen, and endoxifen, respectively ${ }^{40-42}$ ). Decreased plasma concentrations of tamoxifen's lipophilic metabolite $N$ desmethyltamoxifen in patients with high BMIs compared with patients with low BMIs have been reported before ${ }^{8}$ and no influence of body weight on endoxifen formation and endoxifen clearance was determined in our extended covariate analysis, supporting the latter hypothesis.

Our dose escalation simulations for young overweight patients clearly demonstrated that $40 \mathrm{mg}$ tamoxifen q.d. were more adequate for gIMs, reducing the number of patients with subtarget
$\mathrm{C}_{\mathrm{SS}, \text { min ENDX }}$ to $14.2 \%$ in SU1. However, $44.7 \%$ of young overweight gIMs were still at risk in SU2. Moreover, $40 \mathrm{mg}$ and even $60 \mathrm{mg}$ tamoxifen q.d. were not enough to reduce the number of young overweight gPMs with subtarget $\mathrm{C}_{\text {SS,min ENDX }}$ below $36.2 \%$ and $74.4 \%$ in SU1 and SU2, respectively. From this, it follows that other treatment options, like aromatase inhibitors with ovarian function suppression, should be used for young overweight gPMs and obese gIMs, which is an alternative supported by prospective clinical data. ${ }^{43}$

Of note, $99 \%$ of the patients in our pooled dataset received $20 \mathrm{mg}$ tamoxifen q.d. Thus, simulated endoxifen concentrations at higher doses rely on the assumption of dose linearity. Moreover, increasing the dose also increases the concentrations of tamoxifen and its primary metabolites, which has, in part, been associated with more frequent adverse events. ${ }^{44}$ Several studies have reported the feasibility and safety of tamoxifen dose escalations up to $120 \mathrm{mg}$ q.d. ${ }^{45-47}$ However, sample sizes were small and further information on the safety of increased tamoxifen doses has to be generated before their use can be recommended in clinical routine.

Importantly, whereas CYP2D6 AS, body weight, and age explained general trends within the population, the IIV in both tamoxifen clearance (39.9\% coefficient of variation, RSE: $3 \%$ ) and endoxifen formation ( $46 \%$ coefficient of variation, RSE: $3 \%$ ) remained high. Thus, individual $\mathrm{C}_{S S, \text { min ENDX }}$ may deviate from the predictions for typical patients. Moreover, we demonstrated in SU2 that individual risks for subtarget $\mathrm{C}_{\mathrm{SS}, \min \text { ENDX }}$ can largely differ from the average expected risk of the respective typical patient of a specific subpopulation and strongly depend on patients' individual covariate combination. Using a fixed dose could thus lead to subtarget $\mathrm{C}_{S S, \min \text { ENDX }}$ (in case of (young) obese patients) 
but also unnecessary high doses (in case of (elderly) low weight patients, for whom we have found in our previous work ${ }^{22}$ that doses lower than $20 \mathrm{mg}$ q.d. would be sufficient as well.

We, therefore, strongly advocate to use model-informed precision dosing to identify personaliszd tamoxifen doses for $\mathrm{C}_{S S \text {,min }}$ ENDX target attainment ${ }^{22}$ : Based on a patient's CYP2D6 AS, age, and body weight, our model can guide initial dose selection and, if needed, dose refinement upon availability of measured $\mathrm{C}_{\mathrm{SS}, \mathrm{min}}$ ENDX. In this respect, it should be mentioned that the endoxifen target threshold used in this study is yet controversial. However, a recent report from a prospective clinical trial suggesting no relationship between CYP2D6 genotype or $\mathrm{C}_{S S \text {, min } \mathrm{ENDX}}$ and treatment outcome ${ }^{15}$ provoked large criticism with regard to applied methods ${ }^{18,48,49}$ and low statistical power. ${ }^{17}$ Thus, a properly designed and well-powered prospective clinical trial ${ }^{17}$ is needed to assess the relationship between CYP2D6 genotype or $\mathrm{C}_{S S \text {,min }}$ ENDX and breast cancer outcome. Provided the threshold or a similar clinical concentration cut-off point for endoxifen will be confirmed, a patient's CYP2D6 genotype, body weight, and age should be considered in an individualized dose selection process to reach therapeutic endoxifen levels.

\section{SUPPORTING INFORMATION}

Supplementary information accompanies this paper on the Clinical Pharmacology \& Therapeutics website (www.cpt-journal.com).

\section{ACKNOWLEDGMENT}

The authors thank the High-Performance Computing Service of ZEDAT at Freie Universitaet Berlin (https://www.zedat.fu-berlin.de/HPC/Home) for computing time. Open access funding enabled and organized by Projekt DEAL.

\section{FUNDING}

This work was, in part, supported by the Robert Bosch Stiftung, the Bundesministerium für Bildung und Forschung (BMBF; 01EK1509A), and the Deutsche Forschungsgemeinschaft (DFG; MU 1727/2-1 and SCHR 1323/2-1), Germany.

\section{CONFLICT OF INTEREST}

C.K. and W.H. report grants from an industry consortium (AbbVie Deutschland GmbH \& Co. K.G., Boehringer Ingelheim Pharma GmbH \& Co. K.G., Grünenthal GmbH, Astra Zeneca, F. Hoffmann-La Roche Ltd, Merck KGaA and Sanofi) for the PharMetrX program. C.K. reports a grant for the Innovative Medicines Initiative-Joint Undertaking ("DDMoRe"). C.K. and R.Mi. report grants from the Federal Ministry of Education and Research within the Joint Programming Initiative on Antimicrobial Resistance Initiative (JPIAMR), all outside the submitted work. All other authors declared no competing interests for this work.

\section{AUTHOR CONTRIBUTIONS}

A.M. wrote the manuscript. A.M., A.T., B.C., C.K., D.E., E.C., H.B., L.K., M.J., M.S., N.Z., P.N., R.Ma., R.Mi., S.C., S.K., T.M., W.H., and W.S. designed the research. A.M., A.T., B.C., C.K., D.E., E.C., H.B., L.K., M.J., M.S., N.Z., P.N., R.Ma., R.Mi., S.C., S.K., T.M., W.H., and W.S. performed the research. A.M. and L.K. analyzed the data.

(c) 2020 The Authors. Clinical Pharmacology \& Therapeutics published by Wiley Periodicals LLC on behalf of American Society for Clinical Pharmacology and Therapeutics.

This is an open access article under the terms of the Creative Commons Attribution-NonCommercial-NoDerivs License, which permits use and distribution in any medium, provided the original work is properly cited, the use is non-commercial and no modifications or adaptations are made.
1. Cardoso, F.E. et al. Early breast cancer: ESMO Clinical Practice Guidelines for diagnosis, treatment and follow-up†. Ann. Oncol. 30, 1194-1220 (2019).

2. Burstein, H.J. et al. Adjuvant endocrine therapy for women with hormone receptor-positive breast cancer: ASCO clinical practice guideline focused update. J. Clin. Oncol. 37, 423-438 (2019).

3. Abe, 0 . et al. Relevance of breast cancer hormone receptors and other factors to the efficacy of adjuvant tamoxifen: patient-level meta-analysis of randomised trials. Lancet 378, 771-784 (2011).

4. Johnson, M.D. et al. Pharmacological characterization of 4-hydroxy-N-desmethyl tamoxifen, a novel active metabolite of tamoxifen. Breast Cancer Res. Treat. 85, 151-159 (2004).

5. Mürdter, T.E. et al. Activity levels of tamoxifen metabolites at the estrogen receptor and the impact of genetic polymorphisms of phase I and II enzymes on their concentration levels in plasma. Clin. Pharmacol. Ther. 89, 1-10 (2011).

6. Desta, Z. Comprehensive evaluation of tamoxifen sequential biotransformation by the human cytochrome $\mathrm{p} 450$ system in vitro: prominent roles for CYP3A and CYP2D6. J. Pharmacol. Exp. Ther. 310, 1062-1075 (2004).

7. Sanchez-Spitman, A.B. et al. Clinical pharmacokinetics and pharmacogenetics of tamoxifen and endoxifen. Expert Rev. Clin. Pharmacol. 12, 1-14 (2019).

8. Saladores, P. et al. Tamoxifen metabolism predicts drug concentrations and outcome in premenopausal patients with early breast cancer. Pharmacogenomics J. 15, 84-94 (2015)

9. Madlensky, L. et al. Tamoxifen metabolite concentrations, CYP2D6 genotype, and breast cancer outcomes. Clin. Pharmacol. Ther. 89, 718-725 (2011).

10. Schroth, W. et al. Improved prediction of endoxifen metabolism by CYP2D6 genotype in breast cancer patients treated with tamoxifen. Front. Pharmacol. 8, 582 (2017).

11. Puszkiel, A. et al. Factors affecting tamoxifen metabolism in breast cancer patients; preliminary results of the French PHACS study (NCT01127295). Clin. Pharmacol. Ther. 106, 585-595 (2019).

12. Bradley, R. et al. Aromatase inhibitors versus tamoxifen in early breast cancer: patient-level meta-analysis of the randomised trials. Lancet 386, 1341-1352 (2015).

13. Regan, M.M. et al. CYP2D6 genotype and tamoxifen response in postmenopausal women with endocrine-responsive breast cancer: the breast international group 1-98 trial. J. Natl. Cancer Inst. 104, 441-451 (2012).

14. Rae, J.M. et al. CYP2D6 and UGT2B7 genotype and risk of recurrence in tamoxifen-treated breast cancer patients. J. Natl. Cancer Inst. 104, 452-460 (2012).

15. Sanchez-Spitman, A. et al. Tamoxifen pharmacogenetics and metabolism: results from the prospective CYPTAM study. J. Clin. Oncol. 37, 636-646 (2019).

16. Ratain, M.J., Nakamura, Y. \& Cox, N.J. CYP2D6 genotype and tamoxifen activity: understanding interstudy variability in methodological quality. Clin. Pharmacol. Ther. 94, 185-187 (2013).

17. de Vries Schultink, A.H.M.et al.Prospective evaluation of therapeutic drug monitoring of endoxifen: feasibility of observational and randomized trials. PAGE $28<$ http://pagemeeting.org/?abstract=9150> (2019). Accessed October 10, 2019.

18. Braal, C.L. et al. Relevance of endoxifen concentrations: absence of evidence is not evidence of absence. J. Clin. Oncol. 37, 1980-1981 (2019).

19. Wu, A.H. et al. Tamoxifen, soy, and lifestyle factors in Asian American women with breast cancer. J. Clin. Oncol. 25, 30243030 (2007).

20. Peyrade, F. et al. Age-related difference in tamoxifen disposition. Clin. Pharmacol. Ther. 59, 401-410 (1996).

21. Lien, E.A. et al. Serum concentrations of tamoxifen and its metabolites increase with age during steady-state treatment. Breast Cancer Res. Treat. 141, 243-248 (2013).

22. Klopp-Schulze, L. et al. Integrated data analysis of six clinical studies points toward model-informed precision dosing of tamoxifen. Front. Pharmacol. 11, 1-19 (2020). 
23. Antunes, M.V. et al. CYP3A4*22 is related to increased plasma levels of 4-hydroxytamoxifen and partially compensates for reduced CYP2D6 activation of tamoxifen. Pharmacogenomics 16 , 601-617 (2015).

24. Sendur, M.A.N. et al. Effect of body mass index on the efficacy of adjuvant tamoxifen in premenopausal patients with hormone receptor positive breast cancer. J. BUON 21, 27-34 (2016).

25. Pan, H. \& Gray, R.G. Effect of obesity in premenopausal ER+ early breast cancer: EBCTCG data on 80,000 patients in 70 trials. J. Clin. Oncol. 32, 503 (2014).

26. Binkhorst, L. et al. Effects of CYP induction by rifampicin on tamoxifen exposure. Clin. Pharmacol. Ther. 92, 62-67 (2012).

27. Binkhorst, L. et al. Augmentation of endoxifen exposure in tamoxifen-treated women following SSRI switch. Clin. Pharmacokinet. 55, 249-255 (2016).

28. de Graan, A.-J.- M. et al. Dextromethorphan as a phenotyping test to predict endoxifen exposure in patients on tamoxifen treatment J. Clin. Oncol. 29, 3240-3246 (2011).

29. Neven, P. et al. Tamoxifen metabolism and efficacy in breast cancer: a prospective multicenter trial. Clin. Cancer Res. 24 2312-2318 (2018).

30. Poppe, A. et al. Abstract P3-07-46: CYPTAM-BRUT 3: endometrial thickness cannot be used as a marker for tamoxifen metabolization in postmenopausal breast cancer patients. Cancer Res. 76, P3-07-46 (2016).

31. Goetz, M.P. et al. Clinical Pharmacogenetics Implementation Consortium (CPIC) Guideline for CYP2D6 and tamoxifen therapy. Clin. Pharmacol. Ther. 103, 770-777 (2018).

32. Caudle, K.E. et al. Standardizing CYP2D6 genotype to phenotype translation: consensus recommendations from the clinical pharmacogenetics implementation consortium and Dutch Pharmacogenetics Working Group. Clin. Transl. Sci. 13, 116-124 (2020).

33. Pinkerton, J.A.V. et al. The 2017 hormone therapy position statement of the North American Menopause Society. Menopause 24, 728-753 (2017).

34. Ahmad, A. et al. Endoxifen, a new cornerstone of breast cancer therapy: demonstration of safety, tolerability, and systemic bioavailability in healthy human subjects. Clin. Pharmacol. Ther. 88, 814-817 (2010).

35. Sheiner, L.B. \& Beal, S.L. Some suggestions for measuring predictive performance. J. Pharmacokinet. Biopharm. 9, 503-512 (1981).

36. Mueller-Schoell, A.et al.Computational treatment simulations to assess the risk for non-efficacy in tamoxifen treatment for breast cancer patients of different ethnicities. Annual Meeting Deutsche Pharmazeutische Gesellschaft (DPhG), Heidelberg, Germany. September 1-4, 2019 <https://www.dphg.de/fileadmin/downl oads/DPhG-ConferenceBook_2019.pdf> (2019). Accessed December 12, 2019.

37. Jonsson, E.N. \& Karlsson, M.O. Automated covariate model building within NONMEM. Pharm. Res. 15, 1463-1468 (1998).

38. Owen, J.S. \& Fiedler-Kelly, J. Introduction to population pharmacokinetic/pharmacodynamic analysis with nonlinear mixed effects models (John Wiley \& Sons Ltd, Hoboken, NJ, 2014).

39. Cheymol, G. Clinical pharmacokinetics of drugs in obesity: an update. Clin. Pharmacokinet. 25, 103-114 (1993).

40. PubChem Compound summary: Tamoxifen.PubChem <https:// pubchem.ncbi.nlm.nih.gov/compound/10090750> (2019). Accessed December 19, 2019.

41. PubChem Compound Summary: N-Desmethyltamoxifen.PubChem <https://pubchem.ncbi.nlm.nih.gov/compound/N-Desmethylt amoxifen> (2019). Accessed December 19, 2019.

42. PubChem Compound summary: Endoxifen.PubChem <https:// pubchem.ncbi.nlm.nih.gov/compound/10090750> (2019). Accessed December 16, 2019.

43. Francis, P.A. et al. Adjuvant ovarian suppression in premenopausal breast cancer. N. Engl. J. Med. 372, 436-446 (2014).

44. Gallicchio, L. et al. Association of tamoxifen (TAM) and TAM metabolite concentrations with self-reported side effects of TAM in women with breast cancer. Breast Cancer Res. Treat. 85, 89-97 (2004).

45. Dezentjé, V.O. et al. CYP2D6 genotype- and endoxifen-guided tamoxifen dose escalation increases endoxifen serum concentrations without increasing side effects. Breast Cancer Res. Treat. 153, 583-590 (2015)

46. Fox, P. et al. Dose escalation of tamoxifen in patients with low endoxifen level: evidence for therapeutic drug monitoring - the TADE study. Clin. Cancer Res. 22, 3164-3171 (2016).

47. Kiyotani, K. et al. Dose-adjustment study of tamoxifen based on CYP2D6 genotypes in Japanese breast cancer patients. Breast Cancer Res. Treat. 131, 137-145 (2012).

48. Brauch, H., Schroth, W., Mürdter, T. \& Schwab, M. Tamoxifen pharmacogenetics and metabolism: the same is not the same. J. Clin. Oncol. 37, 1981-1982 (2019).

49. Goetz, M.P. et al. Tamoxifen metabolism and breast cancer recurrence: a question unanswered by CYPTAM. J. Clin. Oncol. 37 , 1982-1983 (2019) 